\title{
Correction to: A skeleton-based process planning framework for support-free $3+2$-axis printing of multi-branch freeform parts
}

\section{Xiangyu Wang ${ }^{1} \cdot$ Lufeng Chen $^{2} \cdot$ Tak-Yu Lau $^{1} \cdot$ Kai Tang ${ }^{1}$}

Published online: 23 August 2020

(C) Springer-Verlag London Ltd., part of Springer Nature 2020

Correction to: The International Journal of Advanced Manufacturing Technology.

https://doi.org/10.1007/s00170-020-05790-0

The original published article contained a mistake.

The organization of authors including Xiangyu Wang, Tak$\mathrm{Yu}$ Lau, and Kai Tang is Hong Kong University of Science and Technology, and the organization of author Lufeng Chen is University of Electronic Science and Technology of China.

The correct affiliations are now correctly shown below.

Publisher's note Springer Nature remains neutral with regard to jurisdictional claims in published maps and institutional affiliations.

The online version of the original article can be found at https://oi.org/ 10.1007/s00170-020-05790-0

\footnotetext{
Kai Tang

mektang@ust.hk

Xiangyu Wang

xwangbn@connect.ust.hk

Lufeng Chen

lchenar@connect.ust.hk

Tak-Yu Lau

tylauae@connect.ust.hk

1 Department of Mechanical and Aerospace Engineering, Hong Kong University of Science and Technology, Clear Water Bay, Kowloon, Hong Kong

2 School of Automation Engineering, University of Electronic Science and Technology of China, No. 2006, Xiyuan Ave., West High-tech Zone, Chengdu, Sichuan, China
} 